\title{
Comparison of oxycodone and fentanyl for postoperative patient-controlled analgesia after laparoscopic gynecological surgery
}

\author{
Joong-Ho Park, Chiu Lee, Youngmin Shin, Ji-Hyun An, Jong-Seouk Ban, \\ and Ji-Hyang Lee
}

Department of Anesthesiology and Pain Medicine, Daegu Fatima Hospital, Daegu, Korea

\begin{abstract}
Background: Opioids are widely used in boluses and patient-controlled analgesia (PCA) for postoperative pain control. In this study, we compared the effects of oxycodone and fentanyl on postoperative pain in patients with intravenous patient-controlled analgesia (IV-PCA) after laparoscopic gynecological surgery.

Methods: Seventy-four patients undergoing elective total laparoscopic hysterectomy or laparoscopic myomectomy were randomly assigned to the administration of either fentanyl or oxycodone using IV-PCA (potency ratio $1: 60$ ). The cumulative dose administered in the patient-controlled mode during the initial 48 hours after the operation was measured. Patients were also assessed for postoperative pain severity, adverse effects, and patient satisfaction.

Results: No significant differences were observed in patient satisfaction with the analgesia during the postoperative period. Patients in the oxycodone group experienced significantly more dizziness compared to the fentanyl group. Patients in the oxycodone group showed significantly lower consumption of opioid in the patient-controlled mode $(10.1 \pm 8.5 \mathrm{ml}$ vs. $16.6 \pm 12.0 \mathrm{ml}, \mathrm{P}=0.013)$.

Conclusions: Our data suggest that oxycodone and fentanyl demonstrated similar effects, and therefore oxycodone may be a good alternative to fentanyl in postoperative pain management. Further studies in various clinical settings will be needed to determine the adequate potency ratio.
\end{abstract}

Key Words: Fentanyl, Oxycodone, Patient-controlled analgesia, Postoperative pain.

Corresponding author: Ji-Hyun An, M.D.

Department of Anesthesiology and Pain Medicine, Daegu Fatima

Hospital, 99, Ayang-ro, Dong-gu, Daegu 701-724, Korea

Tel: 82-53-940-7434, Fax: 82-53-954-7417

E-mail: anji43@naver.com

Received: September 19, 2014.

Revised: 1st, October 16, 2014; 2nd, October 17, 2014.

Accepted: October 21, 2014.

Korean J Anesthesiol 2015 April 68(2): 153-158

http://dx.doi.org/10.4097/kjae.2015.68.2.153

\section{Introduction}

For postoperative pain management, opioids are widely used in boluses or with patient-controlled analgesia (PCA). Morphine, fentanyl, and oxycodone are some of the widely used opioids for postoperative pain control. Oxycodone, a semisynthetic thebaine derivative $\mu$-opioid receptor agonist, is replacing morphine as a first-choice opioid in several countries [1]. Oxycodone has been used for the management of cancer-related pain as well as for chronic non-cancer related problems, so controlled

(c) This is an open-access article distributed under the terms of the Creative Commons Attribution Non-Commercial License (http://creativecommons.org/ licenses/by-nc/3.0/), which permits unrestricted non-commercial use, distribution, and reproduction in any medium, provided the original work is properly cited. 
studies have been performed in areas including cancer pain, osteoarthritis-related pain, neuropathic pain, and postoperative pain [2].

There are various studies comparing the effects of oxycodone and morphine on postoperative pain control [3-5]. However, there are few studies regarding the efficacy and the side effects of oxycodone in IV-PCA usage, and there are even fewer studies comparing the effects of oxycodone and fentanyl on postoperative pain [6,7].

In this randomized prospective double-blind study, we compared oxycodone and fentanyl on the basis of adequacy of postoperative pain control using IV-PCA in patients who underwent laparoscopic gynecological operations. We also investigated the occurrence of adverse effects, opioid consumption, and the overall satisfaction of patients with postoperative analgesia.

\section{Materials and Methods}

Prior to conducting the study, we obtained approval from our Institutional Review Board. Written informed consent was obtained from 74 female patients scheduled to undergo either elective total laparoscopic hysterectomy (TLH) or laparoscopic myomectomy under general anesthesia. All patients enrolled in our study were of American Society of Anesthesiologists (ASA) physical status I or II and aged between 20 and 60 years. Patients were randomly assigned to two groups receiving either oxycodone (group O) or fentanyl (group F) for postoperative IV-PCA. Exclusion criteria consisted of history of underlying psychological disorders, chronic pain with history of opioid usage, communication disability, lack of ability to use PCA, and pregnancy. Patients were randomized to either group $\mathrm{O}$ or group $\mathrm{F}$ by a randomization table using Excel (Microsoft Corp., Seoul, Korea).

For premedication, all patients received $0.2 \mathrm{mg}$ of glycopyrrolate intramuscularly 30 minutes prior to surgery. Upon arrival in the operating room, all patients were continuously monitored with electrocardiography, pulse oximetry, and capnography. General anesthesia was induced with $2 \mathrm{mg} / \mathrm{kg}$ of propofol and a remifentanil infusion of $20 \mu \mathrm{g} / \mathrm{min}$. A dose of $0.6 \mathrm{mg} / \mathrm{kg}$ of rocuronium was administered for muscle relaxation prior to intubation. Desflurane was used with nitrous oxide (50\%) for maintenance of anesthesia, and a remifentanil infusion of 5-20 $\mu \mathrm{g} / \mathrm{min}$ was used for analgesia. A dose of $0.075 \mathrm{mg}$ of palonosetron was administered intravenously and another $0.075 \mathrm{mg}$ of palonosetron was mixed into the IV-PCA for prevention of potential postoperative nausea and vomiting (PONV). Following completion of skin closure, desflurane and remifentanil were discontinued. Neuromuscular blockade was reversed with 0.2 $\mathrm{mg}$ of glycopyrrolate and $10 \mathrm{mg}$ of pyridostigmine. Tracheal extubation was performed after recovery of response to verbal commands and spontaneous respiration.
After completion of the operation, patients were transferred to the postanesthetic care unit (PACU), where they continued to be monitored with electrocardiography, pulse oximetry, and capnography. All patients were observed in the PACU for 1 hour before being transferred to the general ward. The patients were connected to the PCA pump (AutoMed $3400^{\circledR}$, Ace Medical, Seoul, Korea) for postoperative pain control. Patients in group $\mathrm{O}$ were assigned to IV-PCA using oxycodone, with a basal infusion of $0.9 \mathrm{mg} / \mathrm{h}$, bolus dose of $0.9 \mathrm{mg}$, and lockout interval of 15 minutes. On the other hand, patients in group F were administered IV-PCA using fentanyl, with a basal infusion of 15 $\mu \mathrm{g} / \mathrm{h}$, bolus dose of $15 \mu \mathrm{g}$, and lockout interval of 15 minutes. Since the units of measure between the two opioids differ, we administered them in a potency ratio of $1: 60$. In the initial pilot study performed in 20 patients, 10 patients were assigned to the usage of oxycodone and 10 to fentanyl, with an initial potency ratio of $1: 100$ (fentanyl : oxycodone). However, 5 in 10 patients receiving oxycodone experienced moderate to severe nausea, vomiting, and dizziness, compared to 2 in 10 patients in the fentanyl group. We continued the pilot study with the potency ratio modified to $1: 80$. Because 4 in 10 patients receiving oxycodone had complaints of nausea and vomiting, however, the potency ratio was changed to $1: 60$ in the final study. When patients complained of severe pain, either oxycodone $2 \mathrm{mg}$ or fentanyl $50 \mu \mathrm{g}$ were administered in IV boluses as rescue analgesics, depending on the group the patients were assigned to. Since there were no previous studies comparing the dosages of rescue analgesics of intravenous bolus oxycodone and fentanyl, the dosages of rescue analgesics were given as recommended by the product information (oxycodone $2 \mathrm{mg}$, fentanyl 50 to $100 \mu \mathrm{g}$ ). For prevention of potential PONV, $0.075 \mathrm{mg}$ of palonosetron was mixed into all PCA devices. When patients developed moderate to severe nausea or vomiting in the PACU, a rescue antiemetic of $10 \mathrm{mg}$ of metoclopramide was administered. All medications administered in the PACU were recorded and this information was maintained as part of the study data.

Monitoring and interviewing of the patients were performed by doctors who were not involved in the study. Evaluations for pain severity and adverse effects were performed at 1, 6, 24, and 48 hours postoperatively. Pain scores were assessed in two categories, at rest and with movement. Pain with movement was defined as pain observed in activities including lifting limbs, sitting, rolling, or coughing. The severity of the pain was evaluated using the visual analogue scale (VAS), with scores ranging from 0 (no pain) to 100 (worst pain imaginable). The occurrence of adverse effects such as nausea, vomiting, dizziness, headache, and sedation was observed and recorded. Patients were asked to evaluate their degree of overall satisfaction with the postoperative analgesia on a 5 -point scale ( $1=$ very unsatisfied, $2=$ unsatisfied, $3=$ neutral, $4=$ satisfied, or $5=$ very satisfied). The cumu- 
lative dose administered in patient-controlled mode (PCM) was recorded in milliliters.

Based on the pilot studies, we estimated the sample size to detect differences in VAS scores with mean differences between the groups, with a power of $80 \%$ and $\alpha=0.05$. The power analysis indicated that a sample size of 32 per group was required to detect significant differences in VAS scores of more than 10 between group $\mathrm{O}$ and group $\mathrm{F}$ with a standard deviation of 14 for each group in the preliminary test. Seventy-four patients were required to allow for possible incomplete data collection or patient dropout. For statistical analysis of the collected data, SPSS 18.0 (SPSS Inc., Chicago, IL, USA) was used. Data were analyzed using the student's t-test for continuous variables with nearly normal distribution, such as patients' information regarding age, weight, height, and opioid consumption. The Mann-Whitney Utest was used for analyzing VAS scores and patients' satisfaction with analgesia. Categorical variables such as ASA classification, type of operation, and adverse effects were analyzed using the chi-square test and Fisher's exact test, as appropriate. Statistical significance was defined as a $\mathrm{P}$ value of less than 0.05 .

Table 1. Demographic and Clinical Characteristics

\begin{tabular}{lcc}
\hline & $\begin{array}{c}\text { Group O } \\
(\mathrm{n}=37)\end{array}$ & $\begin{array}{c}\text { Group F } \\
(\mathrm{n}=32)\end{array}$ \\
\hline Age (yr) & $46.5 \pm 8.6$ & $46.8 \pm 9.4$ \\
Weight (kg) & $62.0 \pm 9.3$ & $59.8 \pm 9.7$ \\
Height (cm) & $159.9 \pm 5.3$ & $157.4 \pm 5.7$ \\
ASA (I/II) & $28 / 9$ & $18 / 14$ \\
Type of operation & $23 / 14$ & $25 / 7$ \\
(TLH/Laparoscopic myomectomy) & & \\
\hline
\end{tabular}

Values are expressed as mean \pm SD. There were no significant differences between the two groups.

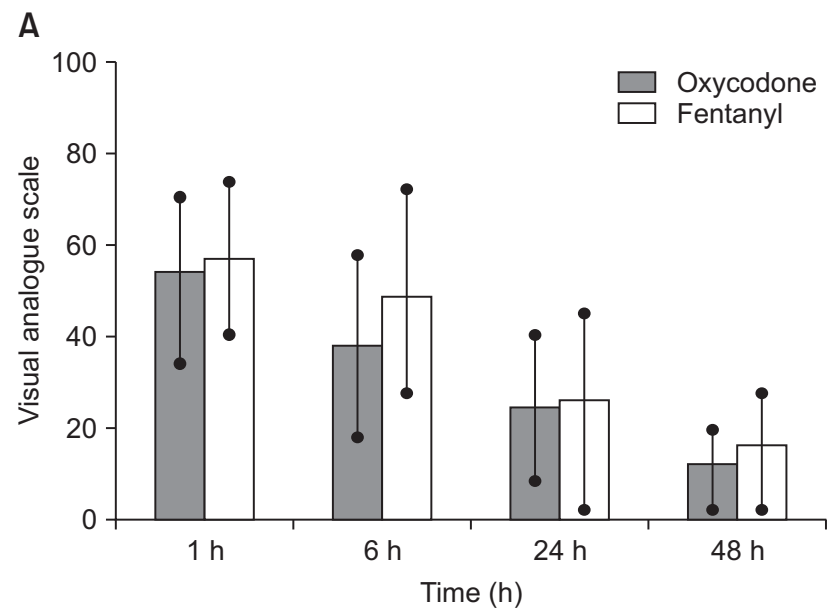

\section{Results}

Among a total of 74 cases of patients scheduled for TLH or laparoscopic myomectomy, the patients were randomly assigned to use either oxycodone (group O) or fentanyl (group F) for postoperative pain control. Initially, 37 patients were assigned to each of the two groups. Of the 37 patients in group F, however, a total of five patients were ultimately excluded from the study, as three patients discontinued the usage of PCA due to severe postoperative nausea, vomiting, and dizziness, while two patients were excluded since the operation was shifted from TLH into open laparotomy. Therefore, a total of 69 patients underwent the final analysis, with 37 patients assigned to group $\mathrm{O}$, and 32 patients assigned to group $\mathrm{F}$.

There were no significant differences in demographic and clinical characteristics including age, height, weight, ASA classification, and the type of operation between the two groups (Table 1).

Cumulative PCA dose in PCM was measured for both groups. It was shown that oxycodone consumption was significantly lower than fentanyl consumption $(10.1 \pm 8.5 \mathrm{ml}$ vs. $16.6 \pm 12.0 \mathrm{ml}$, $\mathrm{P}=0.013)$. Overall, there were no significant differences regarding VAS scores (Fig. 1), with the exception of moving VAS 6 hours postoperatively, which was significantly lower in group $\mathrm{O}$ compared with group $\mathrm{F}(\mathrm{P}=0.008)$.

Adverse events included nausea, vomiting, dizziness, headache, and sedation. Each of the adverse events was evaluated for both opioid groups at 1, 6, 24, and 48 hours postoperatively. Our results showed that patients in group $\mathrm{O}$ experienced more dizziness at 24 hours postoperatively compared to group $\mathrm{F}(\mathrm{P}=0.015)$. The total occurrences of dizziness during the 48 hours were also significantly higher in group $\mathrm{O}(\mathrm{P}=0.020)$. Otherwise, no statistically significant differences between the two groups were

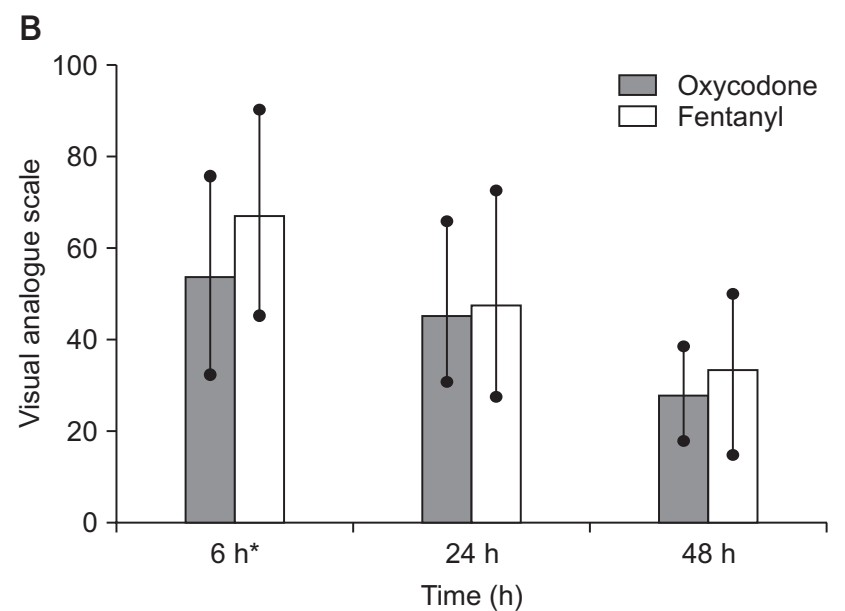

Fig. 1. Resting visual analogue scale (VAS) (A) and moving VAS (B) scores at 1, 6, 24, and 48 hours postoperatively. Moving VAS at 6 hours was significantly lower in the oxycodone group compared with the fentanyl group $(\mathrm{P}=0.008)$. 
Table 2. Incidence of Postoperative Adverse Events during $48 \mathrm{~h}$

\begin{tabular}{lccc}
\hline Adverse event & Oxycodone $(\mathrm{n}=37)$ & Fentanyl $(\mathrm{n}=32)$ & P value \\
\hline Nausea & 11 & 4 & NS \\
Vomiting & 3 & 1 & NS. \\
Dizziness & 13 & 3 & 0.020 \\
Headache & 7 & 1 & NS \\
Sedation & 14 & 10 & NS \\
\hline
\end{tabular}

Values indicate the number of patients. The occurrence of dizziness was significantly higher in group $\mathrm{O}$ compared with group $\mathrm{F}(\mathrm{P}=0.020)$. The patients in group $\mathrm{O}$ experienced more dizziness at $24 \mathrm{~h}$ after the operation compared to the fentanyl group according to the chi-square test and Fisher's exact test $(P=0.015)$. NS: statistically not significant $(\mathrm{P}>0.05)$.

observed regarding the occurrence of adverse events (Table 2).

Patient satisfaction measured at 6 hours postoperatively was significantly higher in group $\mathrm{O}$ compared to group $\mathrm{F}(\mathrm{P}=0.004)$. Other results showed no statistically significant differences regarding patient satisfaction with analgesia (Table 3).

Opioids used for pain control in the PACU were considerably difficult to directly compare, as the unit of measure between the two groups are different from each other. However, under the hypothesis that the effects are similar under the potency ratio of $1: 60$ (fentanyl : oxycodone), the values were input in the program as "fentanyl usage $=$ oxycodone dosage/60", and the two groups were compared using the student's t-test. There were no statistically significant differences observed between the two groups $(\mathrm{P}=0.595)$.

\section{Discussion}

Oxycodone was derived from thebaine in 1916, and has been in clinical usage since 1917 [8]. Today, oxycodone is usually used for the management of chronic pain [2] in controlledrelease tablets. It has been administered through various routes such as intravenous [9,10], intramuscular [11], intranasal [10], subcutaneous [12], rectal [13], epidural [14], and oral routes [11].

Intravenous oxycodone is an effective treatment for acute postoperative pain [15]. In a study by Silvasti et al. [4], similar amounts of morphine and oxycodone were required for sufficient analgesia in patients using IV-PCA for postoperative pain relief for corrective breast or lumbar spinal surgery. In a study by Lenz et al. [3], similar IV-PCA opioid consumptions, pain scores, and adverse effects were found in patients randomly assigned to oxycodone or morphine for postoperative pain control following laparoscopic hysterectomy.

In this study, we compared the postoperative analgesic efficacy, adverse events, opioid consumption, and patient satisfaction of oxycodone and fentanyl in patients scheduled for total laparoscopic hysterectomy or laparoscopic myomectomy. Lenz et al. [3] have compared the effects of oxycodone and morphine on
Table 3. Patient Satisfaction

\begin{tabular}{lccc}
\hline & Oxycodone $(\mathrm{n}=37)$ & Fentanyl $(\mathrm{n}=32)$ & P value \\
\hline $1 \mathrm{~h}$ & $3.3 \pm 1.0$ & $3.1 \pm 1.0$ & $\mathrm{NS}$ \\
$6 \mathrm{~h}$ & $3.9 \pm 1.0$ & $3.2 \pm 1.1$ & 0.004 \\
$24 \mathrm{~h}$ & $4.2 \pm 0.9$ & $3.9 \pm 1.2$ & $\mathrm{NS}$ \\
$48 \mathrm{~h}$ & $4.7 \pm 0.7$ & $4.4 \pm 1.0$ & $\mathrm{NS}$ \\
\hline
\end{tabular}

Values are expressed as mean \pm SD. The $6 \mathrm{~h}$ patient satisfaction of group $\mathrm{O}$ was significantly higher than that of group $\mathrm{F}$. The data was analyzed using the Mann-Whitney U test. NS: statistically not significant $(\mathrm{P}>0.05)$.

patients undergoing laparoscopic hysterectomy. However, studies comparing the effects of oxycodone and fentanyl are rare. Koch et al. [6] have compared the effects of intravenous oxycodone and fentanyl on postoperative visceral pain after outpatient laparoscopic cholecystectomy, with the finding that oxycodone provided better analgesia but also more side effects. Hwang et al. [7] have insisted that oxycodone may be useful as an alternative to fentanyl for PCA following laparoscopic cholecystectomy. This, to our knowledge, is the only previous study comparing the effects of PCA mediated oxycodone and fentanyl.

While intravenous oxycodone appears to be equipotent with morphine [13], it has been reported that the potency ratio of fentanyl to morphine has a range of $1: 80-100$ [16]. Hwang et al. [7] have suggested that the potency ratio of fentanyl to oxycodone might be around $1: 75$ and that oxycodone might be more potent than morphine $(4: 3)$. Therefore, we originally conducted this study under the hypothesis that the potency ratio of fentanyl to oxycodone was $1: 80-100$. In the pilot study, the potency ratio of fentanyl to oxycodone was set at $1: 100$. As nausea and vomiting was induced with oxycodone in the pilot study, however, the potency ratio was changed to $1: 80$. In spite of adjustment of the potency ratio, adverse events persisted, and we therefore established the potency ratio between the two opioids as $1: 60$ in the final study, which uses lower doses of oxycodone compared with previous studies [6,7]. Our results showed that the amount of PCA consumption in patient-controlled mode was significantly lower in group $\mathrm{O}$ than in group $\mathrm{F}$. This seems to suggest that oxycodone is more potent than morphine. This finding is in line with previous studies that have also suggested that oxycodone may be more potent than morphine [3,5-7]. Another possibility that our results seem to suggest is, that the potency ratio of fentanyl to oxycodone may be slightly different than previously thought.

The analgesic effect was measured with VAS and patient satisfaction. Patient satisfaction at $6 \mathrm{hr}$ postoperatively was significantly higher, and moving VAS 6 hours after operation was significantly lower in group O compared with group F. Our results seem to suggest that oxycodone could be more efficient compared with fentanyl in postoperative pain control. There are other studies that have also favored the efficacy of oxycodone over 
other opioids. Minami et al. [17] compared the analgesic profiles of morphine, oxycodone, and fentanyl in a mouse pain model, resulting in oxycodone showing distinct analgesic effects compared with the other two opioids. Koch et al. [6] demonstrated that although oxycodone resulted in more side effects compared to fentanyl, it also provided better analgesia. A study by Ashburn et al. [18] showed that immediate-release oxycodone resulted in more rapid onset of analgesia and was well tolerated in comparison with fentanyl buccal tablets in opioid-tolerant patients with chronic pain. However, the efficacy of oxycodone over fentanyl is controversial, and more studies on this subject are necessary for confirmation.

Our results showed that oxycodone produced significantly more dizziness overall compared with fentanyl $(\mathrm{P}=0.020)$. However, statistically significant differences in 1, 6, 24, and 48 hour postoperative dizziness were only observed at 24 hours postoperatively $(\mathrm{P}=0.015)$. Other adverse events such as nausea, vomiting, headache, and sedation showed no statistically significant differences. The precise mechanisms of opioidinduced nausea and vomiting are not known with certainty, although the nausea and vomiting may be due to multiple opioid effects such as enhanced vestibular sensitivity, direct effects on the chemoreceptor trigger zone, and delayed gastric emptying [19]. The dizziness may have been caused by increased vestibular sensitivity, which perhaps might have been caused by opioids activating mu-opioid receptors on the vestibular epithelium [20]. Although other studies [7,21] have shown that oxycodone produced more nausea compared to other opioids, statistically significant differences could not be observed between the two groups regarding the occurrence of nausea in our study. There were three cases in group $\mathrm{F}$ that were excluded from the study since adverse events including nausea, vomiting, and dizziness occurred and the use of PCA was stopped. If these cases had been included in the final study, our results might have shown that fentanyl induces more nausea compared with oxycodone, contrary to the findings of previous studies [7,21]. To confirm this hypothesis, we conducted an analysis including the three excluded cases. The results showed no significant differences between the two groups in nausea, vomiting, and dizziness.

There are some limitations to this study. There were only 69 patients included in our analysis, and there are limited studies comparing the postoperative effects of oxycodone and fentanyl. Therefore, more studies may be necessary regarding this subject. In the current study, the potency ratio of fentanyl to oxycodone was $1: 60$, which is different from the ratios of previous studies. A study conducted using various potency ratios might be of more benefit in determining the adequate potency ratio. Another limitation of this study is that it was limited to female patients in ASA classification I or II. Studies should be conducted for patients with various demographic and clinical characteristics. Additionally, clinical data indicate that not only the type of surgery but also the extent of surgery may affect the comparison of different opioid analgesics [12]. Therefore, studies comparing various types of operations should be performed. Finally, although our observed lack of significant differences in opioid consumption, VAS, patient satisfaction, and adverse effects may suggest that oxycodone may be a good alternative to fentanyl in postoperative IV-PCA, it may be too early to conclude that there are no differences in the occurrences of adverse events. Studies with larger sample sizes may be helpful in drawing more definitive conclusions on this matter.

In conclusion, oxycodone may be a good alternative to fentanyl in postoperative pain management using PCA. Our data suggest that in general, oxycodone showed similar, and sometimes better effects in postoperative analgesia compared with fentanyl. However, further studies in various clinical settings are needed to determine the adequate potency ratio and the efficacy between oxycodone and fentanyl.

\section{References}

1. Choi BH. Oxycodone: a new therapeutic option in postoperative pain management. JKDSA 2013; 13: 167-78.

2. Kalso E. Oxycodone. J Pain Symptom Manage 2005; 29(5 Suppl): S47-56.

3. Lenz H, Sandvik L, Qvigstad E, Bjerkelund CE, Raeder J. A comparison of intravenous oxycodone and intravenous morphine in patientcontrolled postoperative analgesia after laparoscopic hysterectomy. Anesth Analg 2009; 109: 1279-83.

4. Silvasti M, Rosenberg P, Seppälä T, Svartling N, Pitkänen M. Comparison of analgesic efficacy of oxycodone and morphine in postoperative intravenous patient-controlled analgesia. Acta Anaesthesiol Scand 1998; 42: 576-80.

5. Kalso E, Pöyhiä R, Onnela P, Linko K, Tigerstedt I, Tammisto T. Intravenous morphine and oxycodone for pain after abdominal surgery. Acta Anaesthesiol Scand 1991; 35: 642-6.

6. Koch S, Ahlburg P, Spangsberg N, Brock B, Tønnesen E, Nikolajsen L. Oxycodone vs. fentanyl in the treatment of early post-operative pain after laparoscopic cholecystectomy: a randomised double-blind study. Acta Anaesthesiol Scand 2008; 52: 845-50.

7. Hwang BY, Kwon JY, Kim E, Lee DW, Kim TK, Kim HK. Oxycodone vs. fentanyl patient-controlled analgesia after laparoscopic cholecystectomy. Int J Med Sci 2014; 11: 658-62.

8. Sneader WE. Drug discovery: a history. West Sussex. John Wiley \& Sons Inc. 2005, p 119. 
9. Pöyhiä R, Olkkola KT, Seppälä T, Kalso E. The pharmacokinetics of oxycodone after intravenous injection in adults. Br J Clin Pharmacol 1991; 32: 516-8.

10. Takala A, Kaasalainen V, Seppälä T, Kalso E, Olkkola KT. Pharmacokinetic comparison of intravenous and intranasal administration of oxycodone. Acta Anaesthesiol Scand 1997; 41: 309-12.

11. Pöyhiä R, Seppälä T, Olkkola KT, Kalso E. The pharmacokinetics and metabolism of oxycodone after intramuscular and oral administration to healthy subjects. Br J Clin Pharmacol 1992; 33: 617-21.

12. Maddocks I, Somogyi A, Abbott F, Hayball P, Parker D. Attenuation of morphine-induced delirium in palliative care by substitution with infusion of oxycodone. J Pain Symptom Manage 1996; 12: 182-9.

13. Leow KP, Cramond T, Smith MT. Pharmacokinetics and pharmacodynamics of oxycodone when given intravenously and rectally to adult patients with cancer pain. Anesth Analg 1995; 80: 296-302.

14. Backlund M, Lindgren L, Kajimoto Y, Rosenberg PH. Comparison of epidural morphine and oxycodone for pain after abdominal surgery. J Clin Anesth 1997; 9: 30-5.

15. Kokki H, Kokki M, Sjövall S. Oxycodone for the treatment of postoperative pain. Expert Opin Pharmacother 2012; 13: 1045-58.

16. Pereira J, Lawlor P, Vigano A, Dorgan M, Bruera E. Equianalgesic dose ratios for opioids. a critical review and proposals for long-term dosing. J Pain Symptom Manage 2001; 22: 672-87.

17. Minami K, Hasegawa M, Ito H, Nakamura A, Tomii T, Matsumoto M, et al. Morphine, oxycodone, and fentanyl exhibit different analgesic profiles in mouse pain models. J Pharmacol Sci 2009; 111: 60-72.

18. Ashburn MA, Slevin KA, Messina J, Xie F. The efficacy and safety of fentanyl buccal tablet compared with immediate-release oxycodone for the management of breakthrough pain in opioid-tolerant patients with chronic pain. Anesth Analg 2011; 112: 693-702.

19. Smith HS, Laufer A. Opioid induced nausea and vomiting. Eur J Pharmacol 2013; 722: 67-78.

20. Yates BJ, Miller AD, Lucot JB. Physiological basis and pharmacology of motion sickness: an update. Brain Res Bull 1999; 47: 395-406.

21. Pedersen KV, Olesen AE, Drewes AM, Osther PJ. Morphine versus oxycodone analgesia after percutaneous kidney stone surgery: a randomised double blinded study. Urolithiasis 2013; 41: 423-30. 